\title{
Design, service and testing grounds stress spectra and their using to fatigue life assessment of bus bodyworks
}

\author{
Miloslav Kepka ${ }^{1, *}$, Miloslav Kepka Jr. ${ }^{1}$ \\ ${ }^{1}$ University of West Bohemia, Faculty of Mechanical Engineering, Univerzitni 8, 30614 Pilsen, Czech Republic
}

\begin{abstract}
In the development of a road vehicle such as a bus, the input data for the assessment of the fatigue life of the body and other mechanical parts of the vehicle are gradually refined. In the initial phase of the development of a new vehicle, so-called design stress spectra are used. It is necessary to estimate the design spectrum parameters correctly. At the vehicle's prototype testing phase, the so-called service stress spectra can be evaluated. It is essential to determine the characteristic operating modes of the vehicle and to record representative stress-time histories of the critical sections of the construction nodes and vehicle components. Accelerated driving test of fatigue life can be performed on special test routes. The acceleration of such a fatigue test is dependent on the test track composition. The paper generally describes the methodology of such a process and demonstrates it using unique real-time data acquired during the development of a bus.
\end{abstract}

\section{Introduction}

In principle, the following input data are required to assess the fatigue life of the construction nodes of vehicles:

- fatigue life curves of the investigated construction nodes (S-N curves),

- information on their loads in operation (representative stress-time histories in critical points of the investigated nodes).

Stress-time histories are converted to stress spectra (histograms of frequency of harmonic stress cycles).

Calculations of fatigue damage and predictions of fatigue life are based on cumulative damage rules proposed by various authors. The input data are refined during the development of a new vehicle. In the design phase, parameters of $\mathrm{S}-\mathrm{N}$ curves are most often estimated, taken from literature, standards or past solutions. Later, laboratory fatigue tests on structural nodes (which already respect specific structural geometry and manufacturing technology) allow them to determine their corresponding S-N curves.

Unless a mobile vehicle (functional sample, test prototype) is available, it is not possible to perform any strain gauge measurements of service stresses. Therefore, so-called design stress spectra are used in the design phase of vehicles. The design stress spectra are based on empirical experience, sometimes they are part of design standards and regulations, sometimes they represent corporate know-how.

With the vehicle prototype, strain gauge measurements can be made during the vehicle operation on actual lines with various road surface and various unevenness. A differently loaded vehicle (typically empty and fully loaded vehicle) can be measured. By the rain-flow method, the recorded stress-time histories are transformed into so-called service stress spectra.

Measurements should also be carried out on testing grounds. The measurements in this case take place on precisely specified test tracks with precisely given surface roughness. Based on a relative comparison of their damaging effects with fatigue damage generated on real lines, an accelerated running fatigue test of the vehicle can be proposed on testing grounds.

\section{Detail of interest}

The detail of interest was a severely stressed beam joint in the top corner of the door opening in the bus body shown in Fig. 1.

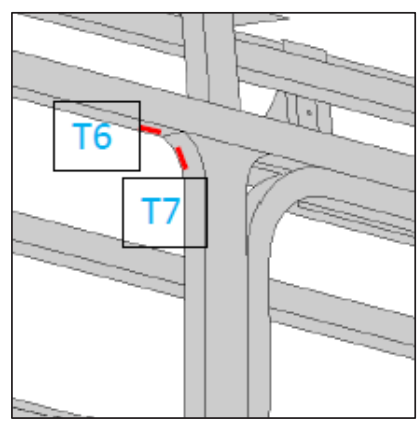

Fig. 1. Detail of interest (T6) - schematic illustration.

The critical cross section was monitored by strain gauge T6. The desired (design) fatigue service life of the vehicle in question was defined $\mathrm{L}_{\mathrm{d}}=1,000,000 \mathrm{~km}$-run. 
This study is a virtual investigation because the specific values used in commercial contracts for various manufacturers are confidential. However, the values and data employed in this study are realistic and can be encountered in real life of a bus or a similar vehicle (trolleybus, battery bus or other vehicle).

\section{S-N curve}

In order to determine the fatigue strength of the evaluated structural detail, laboratory fatigue testing was carried out. Test specimens were equivalent to the considered beam joint in terms of basic material, shape and manufacturing technology (welding).

The transformation of the nominal loading into a root of notch [1] was not necessary, because the approach of the equivalent structural stress was used.

The test specimens were made from thin-walled welded closed profiles, which had $70 \times 50 \mathrm{~mm}$ crosssection and $2 \mathrm{~mm}$ wall thickness and were made of S235JR steel. The critical cross-section of the joint was subjected to reverse bending load (the cycle stress ratio was $\mathrm{R}=-1$ ).

During testing, the stresses acting on the critical cross-section were measured by strain gauges attached approximately $5 \mathrm{~mm}$ from the toe of the fillet weld. The measured values by strain gauges T6 can therefore be referred to as the equivalent structural stress.

The limit state was defined by the instant at which a macroscopic fatigue crack forms (1 to $2 \mathrm{~mm}$ ). In all cases, fatigue cracks initiated in the transition zone of the fillet weld.

Statistical evaluation of the fatigue test data yielded the parameters of the mean S-N curve for the structural detail in the form:

$$
\log \left(N_{f}\right)=14.54-4.53 \cdot \log \left(\sigma_{a}\right) ; \sigma_{c}=60 \mathrm{MPa}
$$

Fig. 2 shows a photograph of the test stand. Table 1 summarises test results. The results of laboratory fatigue tests and the evaluated mean S-N curve are shown in Fig. 3.

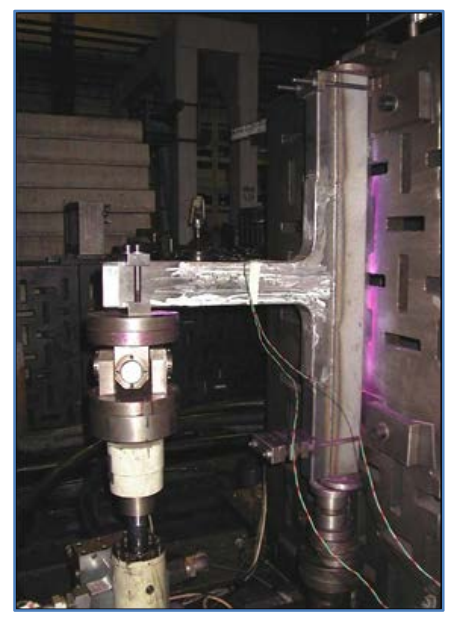

Fig. 2. Test stand.
Table 1. Results of laboratory fatigue tests.

\begin{tabular}{|c|c|c|c|}
\hline $\begin{array}{c}\text { Test } \\
\text { specimen }\end{array}$ & $\begin{array}{c}\text { Testing } \\
\text { stress } \\
\text { amplitude } \\
\sigma_{\mathrm{a}}(\mathrm{Mpa})\end{array}$ & $\begin{array}{c}\text { Limit } \\
\text { fatigue } \\
\text { stage } \\
\mathrm{N}_{\mathrm{f}} \text { (cycles) }\end{array}$ & Remark \\
\hline 1 & 140 & 50,000 & \\
\hline 2 & 120 & 140,000 & \\
\hline 3 & 110 & 170,000 & \\
\hline 4 & 100 & 500,000 & \\
\hline 5 & 80 & $1,250,000$ & \\
\hline 6 & 70 & 900,000 & \\
\hline 7 & 60 & $2,000,000$ & runouts \\
\hline 8 & 50 & $2,000,000$ & runouts \\
\hline
\end{tabular}

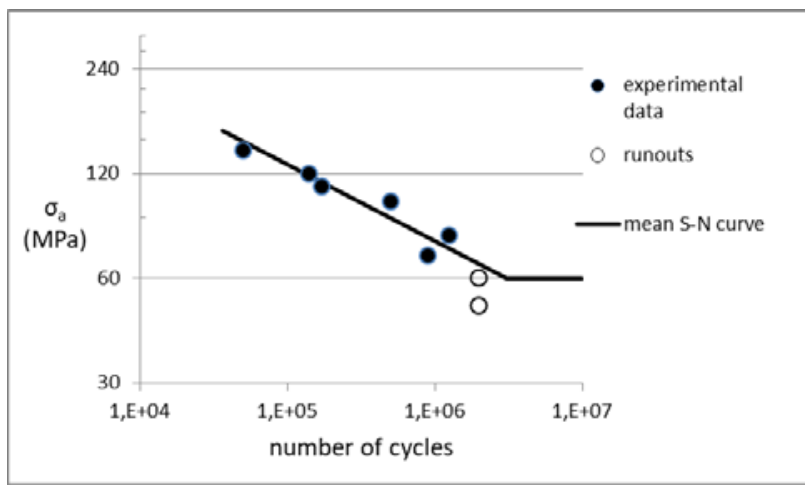

Fig. 3. Results of laboratory fatigue tests and mean S-N curve.

\section{Stress spectra}

\subsection{Design stress spectra}

If accurate data from a measurement is not available, the stress spectra should be estimated. The design stress spectra for this parametric study were generated using the relative coordinates $\sigma_{a i} / \sigma_{a \max }[2]$.

$$
h_{i}=H_{t o t} \cdot\left(\frac{H_{\max }}{H_{\text {tot }}}\right)^{\left(\frac{\sigma_{a i}}{\sigma_{\text {amax }}}\right)^{s}}
$$

$\sigma_{\text {amax }}$ - maximum stress amplitude in the spectrum,

$H_{\text {max }}$ - number of cycles with $\sigma_{\text {amax }}$ amplitude,

$H_{\text {tot }}$ - total number of cycles in the spectrum,

$s \quad$ - shape parameter of the spectrum,

$h_{i} \quad$ - cumulative frequency of cycles with $\sigma_{a i}$ amplitude.

At a constant width of the classification interval $d \sigma_{a}$, one can derive the discrete stress spectrum $\sigma_{a i}-n_{i}$. Absolute class frequency is calculated: $n_{i}=h_{i}-h_{i+1}$, this frequency is assigned to the mid-point of the class (or, safely, to its upper limit) $\sigma_{a i}$. With this interpretation of various oscillation processes, the cumulative frequency distribution of cycles $h_{i}$ is plotted in semi-log graphs using various shape parameters $s$ (Fig. 4): 
$s=\infty$ rectangular distribution for a constantamplitude harmonic process,

$s=1$ normal distribution for a steady-state random Gaussian process with a standard deviation of $\sigma_{R M S}$,

$s=2$ linear distribution for a process consisting of a number of steady-state random Gaussian sub-processes with various values of $\sigma_{R M S}$.

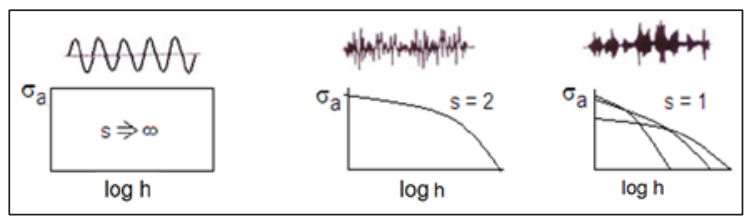

Fig. 4. Characteristic shapes of design stress spectra.

Measurements of service loads in road vehicles were interpreted in literature [3] with the following observations: long-term monitoring of rides on an irregular road surface yields linear distribution stress spectra $(\mathrm{s}=1)$ and manoeuvres (curve riding, braking, etc.) lead to stress spectra with normal distributions $(s=2)$.

An estimate of the total number of cycles $H_{t o t}$ in the design stress spectrum can be obtained from the equation:

$$
H_{t o t}=\frac{L_{d}}{v} \cdot 3,600 \cdot f
$$

$L_{d} \quad$ - design life of the body (in kilometres),

$v \quad$ - average speed (kph),

$f \quad$ - dominant frequency of vibration $(\mathrm{Hz})$.

The frequency $H_{\max }$ of maximum service stress amplitudes $\sigma_{\text {amax }}$ during the design life of the bodywork $L_{d}$ depends on operating conditions of the vehicle given by the user. For the parametric studies, operating condition indicators $P$ and $L_{l}$, were established to indirectly express the severity of the conditions:

$$
P=\frac{H_{\max }}{H_{t o t}}
$$

and

$$
L_{1}=\frac{L_{d}}{H_{\max }}
$$

$P$ - probability of occurrence of the maximum service stress amplitude $\sigma_{\text {amax }}$,

$L_{1}$ - travelled distance (in kilometres), in which the maximum service stress amplitude $\sigma_{\text {amax }}$ occurs once. valid:

Between both indicators the following relationship is

$$
P=\frac{L_{d}}{L_{1} \cdot H_{t o t}}
$$

Therefore, the design stress spectrum parameters $(s$, $H_{t o t}, \sigma_{a \max }$ and $H_{\max }$ ) for designing and sizing the beam joints of the body were selected on the basis of the following considerations.

The major cause of the service loads that act on the structural detail is the vibration of the body due to the vehicle's ride on irregular road surfaces of various quality. For this reason, the shape parameter of the design stress spectrum was chosen as $s=1$.

The design life of the body was set as $L_{d}=10^{6} \mathrm{~km}$. The dominant vibration frequency was assumed to be $f=10 \mathrm{~Hz}$. The average travel speed was set as $v=50 \mathrm{kph}$. After substituting the values into equation (2), the estimation of total number of cycles in the specified body life was found as $H_{t o t}=7.2 \cdot 10^{8}$ cycles.

Maximum stress amplitudes $\sigma_{\text {amax }}$ were estimated by strain gauge measurements on model testing track. The model testing track is usually made from artificial obstacles in a form of cylinder segment having the basis of $500 \mathrm{~mm}$ and height of $60 \mathrm{~mm}$. The artificial obstacles are laid on an even asphalt road in such composition, that overruns of them occurred gradually by right-hand wheels, by both wheels simultaneously and by left-hand wheels [4].

Two loading states (payloads) were measured (run with empty vehicle and run with fully loaded vehicle). The results of the measurements are collected in Table 2.

Table 2. Measurement on model testing track.

\begin{tabular}{|c|c|c|c|c|}
\hline \multicolumn{2}{|c|}{ Empty vehicle } & Right & Both & Left \\
\hline$\sigma_{\text {amax }}$ & $\mathrm{MPa}$ & 45 & 84 & 75 \\
\hline
\end{tabular}

\begin{tabular}{|c|c|c|c|c|}
\hline \multicolumn{2}{|c|}{ Loaded vehicle } & Right & Both & Left \\
\hline$\sigma_{\text {amax }}$ & $\mathrm{MPa}$ & 44 & 108 & 73 \\
\hline
\end{tabular}

As safe values for generation of design stress spectra were finally used the following values: $\sigma_{\text {amax }}=85 \mathrm{MPa}$ for the empty vehicle and $\sigma_{\operatorname{amax}}=110 \mathrm{MPa}$ for the loaded vehicle.

Travelled distance $L_{l}$, in which the maximum stress amplitude $\sigma_{\text {amax }}$ occurs once, was chosen in three alternative variants, $L_{l}=10,100$ and $1000 \mathrm{~km}$, it means with three different values $H_{\max }=10^{5}, 10^{4}$ and $10^{3}$ cycles. The parameters of all alternative design stress spectra are summarised in Table 3. The obtained design stress spectra are plotted and analysed in chapter 3.4.

Table 3. Parameters of alternative design stress spectra.

\begin{tabular}{|c|c|c|c|c|}
\hline \multicolumn{2}{|c|}{ Empty vehicle } & DSS_10_E & DSS_100_E & DSS_1000_E \\
\hline$\sigma_{\text {amax }}$ & $\mathrm{MPa}$ & 85 & 85 & 85 \\
\hline$H_{\max }$ & \multirow{2}{*}{ cycles } & $10^{5}$ & $10^{4}$ & $10^{3}$ \\
\cline { 1 - 4 } & & \multicolumn{3}{|c|}{$7.2 \cdot 10^{8}$} \\
\cline { 1 - 5 }$H_{\text {tot }}$ & - & \multicolumn{3}{|c}{1} \\
\hline
\end{tabular}

\begin{tabular}{|c|c|c|c|c|}
\hline \multicolumn{2}{|c|}{ Loaded vehicle } & DSS_10_L & DSS_100_L & DSS_1000_L \\
\hline$\sigma_{\text {amax }}$ & $\mathrm{MPa}$ & 110 & 110 & 110 \\
\hline$H_{\max }$ & \multirow{2}{*}{ cycles } & $10^{5}$ & $10^{4}$ & $10^{3}$ \\
\cline { 1 - 4 }$H_{\text {tot }}$ & & \multicolumn{3}{|c|}{$7.2 \cdot 10^{8}$} \\
\cline { 1 - 4 }$s$ & - & \multicolumn{3}{|c}{1} \\
\hline
\end{tabular}

These alternative design stress spectra were representations of the potential service at various operating condition indicators $P$ and $L_{l}$. 
In this particular case, the relationship between the operating condition indicators is as follows:

$$
P=\frac{L_{d}}{L_{1} \cdot H_{t o t}}=\frac{1.3889 \cdot 10^{-3}}{L_{1}}
$$

\subsection{Service stress spectra}

If there is a sufficiently representative record of the stress time history, it is more accurate to evaluate the stress spectra from these data. The random processes are converted with the "rain-flow" method into oneparameter or two-parameter histograms of stress cycles depending on the magnitude of their amplitudes and mean values.

The real service stresses are measured by means of strain gauges. In this case, the service stress-time histories were measured for a city bus riding on an irregular surface along a city route whose total length was $L_{m} \approx 40 \mathrm{~km}$.

The signals were analysed in the frequency domain, and insignificant high frequencies were eliminated before using the rain-flow technique. As part of this rainflow step, small cycles with amplitudes below $2 \mathrm{MPa}$ were omitted.

In high-cycle fatigue of welded structures, the mean stress of the cycle does not play a major role. Therefore, only the one-parameter stress spectra $\sigma_{a i}-n_{i}$ were used for subsequent calculations.

Fig. 5 shows procedure of extrapolation of measured stress-time histories into service stress spectra.

The obtained service stress spectra are plotted and analysed in chapter 3.4.

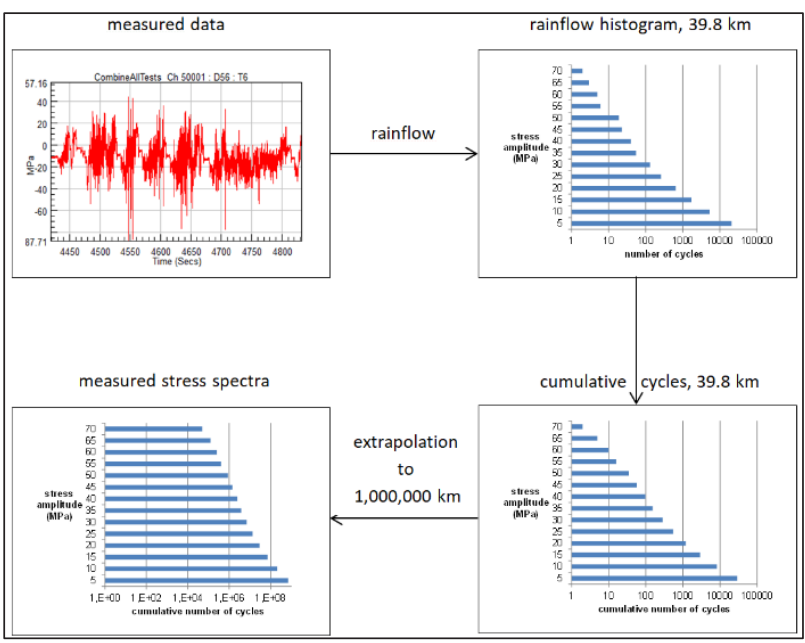

Fig. 5. Extrapolation of measured stress-time history into service stress spectrum (empty vehicle).

\subsection{Testing grounds stress spectra}

In a similar manner to representative urban traffic, the stress spectra on the test polygon were evaluated. The test polygon offers sections, tracks and roads with various longitudinal road profile and different surface quality. Table 4 specifies the composition of the proposed test route.
The measurement was repeated tree times with empty vehicle and three times with fully loaded vehicle. Total length of the measured route was $L_{m} \approx 35.5 \mathrm{~km}$.

The obtained testing grounds stress spectra are plotted and analysed in chapter 3.4.

Table 4. Composition of test route on testing grounds.

\begin{tabular}{|c|c|}
\hline Section & $\mathrm{km}$ \\
\hline slope circuit & 3.80 \\
\hline speed circuit & 2.80 \\
\hline arrival to special roads & 0.07 \\
\hline panel road & 0.45 \\
\hline exit/arrival & 0.13 \\
\hline sinus resonance road & 0.40 \\
\hline exit/arrival & 0.15 \\
\hline paved road & 0.40 \\
\hline exit/arrival & 0.15 \\
\hline paved road & 0,40 \\
\hline exit/arrival & 0.15 \\
\hline paved road & 0.40 \\
\hline exit/arrival & 0.16 \\
\hline Belgian paving & 0.40 \\
\hline exit from special roads & 0.08 \\
\hline speed circuit & 1.90 \\
\hline Total & 11.84 \\
\hline
\end{tabular}

\subsection{Analyses of all stress spectra}

All theoretically generated design stress spectra and all measured service stress spectra and testing grounds stress spectra are plotted and compared in Fig. 6.
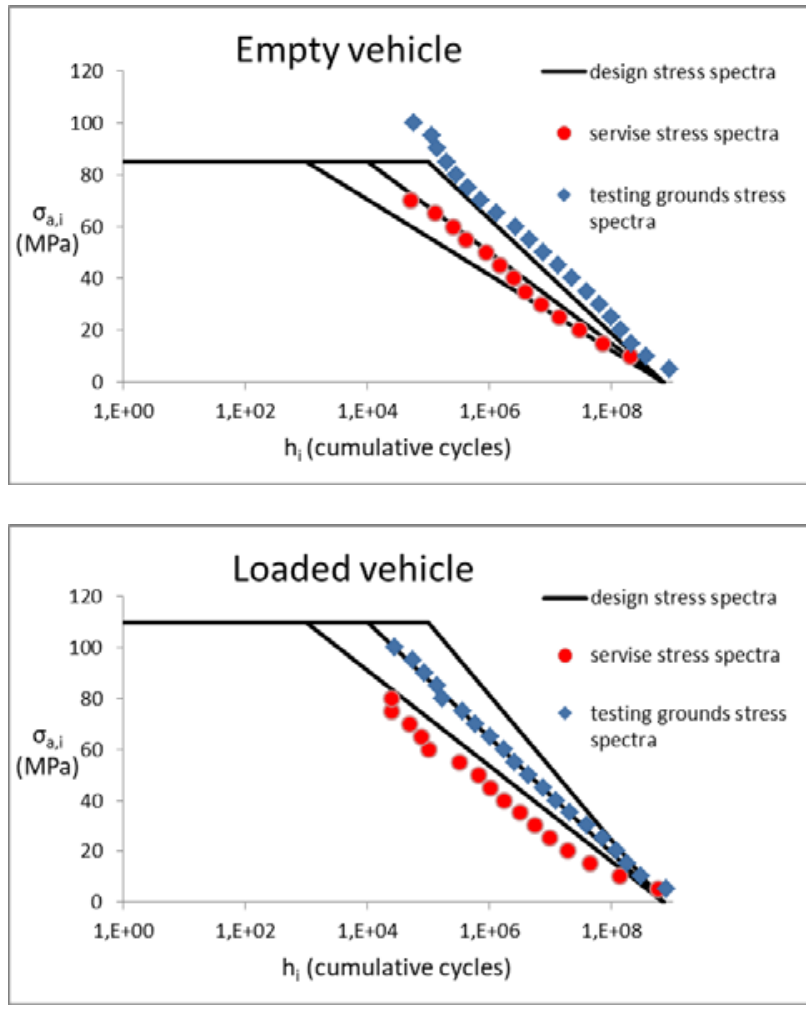

Fig. 6. Stress spectra - empty and fully loaded vehicle. 
The difference in the aggressiveness of the individual spectra we can see at first glance. The shape of all measured stress spectra corresponds well to the assumed linear distribution, too.

In the table 5 there are the values of the shape parameter $s$ from equation of design spectra (1). The values were calculated from experimental data by regression analysis. The shape parameter in all cases approaches to $s \approx 1$.

Table 5. Shape parameter of all measured stress spectra.

\begin{tabular}{|c|c|c|}
\hline $\begin{array}{c}s-\text { shape } \\
\text { parameter }\end{array}$ & $\begin{array}{c}\text { Real } \\
\text { operation }\end{array}$ & $\begin{array}{c}\text { Testing } \\
\text { grounds }\end{array}$ \\
\hline Empty vehicle & 1.011 & 1.092 \\
\hline Loaded vehicle & 0.939 & 1.027 \\
\hline
\end{tabular}

\section{Fatigue life calculations}

The mostly the fatigue damage $D$ is calculated using the linear cumulative damage rule. According to this rule, the limit state with respect to fatigue is reached (i.e. the fatigue life of the structural part is exhausted) when the following condition is met:

$$
D=\sum_{i} \frac{n_{i}}{N_{i}}=D_{l i m}
$$

$D \quad$ - fatigue damage caused by the stress spectrum imposed,

$n_{i} \quad-\quad$ number of cycles applied at the i-th level of stress with the amplitude $\sigma_{\mathrm{ai}}$,

$N_{i} \quad$ - limit life under identical loading (the number of cycles derived from the S-N curve for the part in question at the amplitude $\sigma_{a i}$ ),

$D_{\text {lim }}$ - limit value of fatigue damage.

Various rules apply various boundary conditions to fatigue damage calculation. A schematic representation of these boundary conditions is shown in Fig. 7.

Account is taken of the damage caused by cycles with small amplitudes $\left(\sigma_{a t h}<\sigma_{a i}<\sigma_{c}\right)$, which occur very frequently. A threshold value $\sigma_{a t h}$ is applied to the conversion of stress to damage, and therefore the damage caused by cycles with amplitudes of $\left(\sigma_{a i}<\sigma_{a t h}<\sigma_{c}\right)$ is neglected.

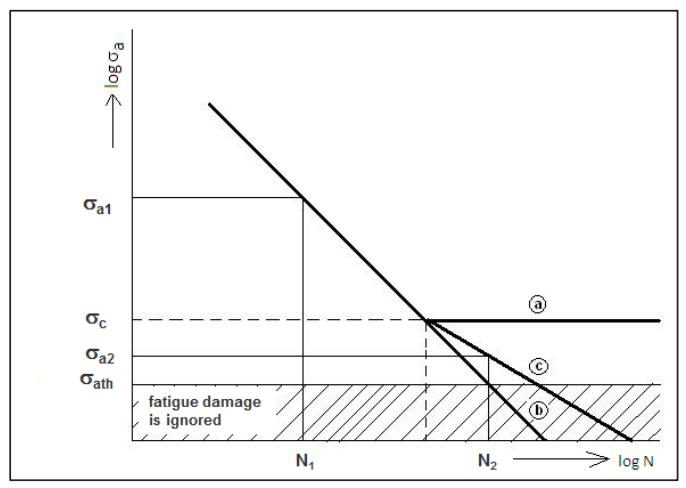

Fig. 7. Boundary conditions for fatigue damage calculations.
A limit value is set for the fatigue damage. According to Miner, $D_{\text {lim }}=1$, but it has been shown experimentally that this value may decline towards hazardous levels with $D_{\text {lim }}<1$.

In the present case, the Haibach-modified version of the Palmgren-Miner rule was chosen for calculating fatigue damage. The limit number of cycles $N_{i}$ was determined as follows:

$$
\text { - } \sigma_{a i} \geq \sigma_{c}: \quad N_{i}=N_{c} \cdot\left(\frac{\sigma_{c}}{\sigma_{a i}}\right)^{w}
$$

and

$$
\text { - } \sigma_{c}>\sigma_{a i} \geq \sigma_{a t h}: \quad N_{i}=N_{c} \cdot\left(\frac{\sigma_{c}}{\sigma_{a i}}\right)^{w d}
$$

Haibach recommends the exponent for the lower part of the S-N curve to be set as $w_{d}=2 w-1$. In this study, the value was $w_{d}=8$. The threshold stress amplitude for taking the fatigue damaging into account was given as $\sigma_{a t h}=0.5 \cdot \sigma_{c}$ in the present case. The limit value of fatigue damage was taken as $D_{\text {lim }}=0.5$.

The computational estimate of service fatigue life (in kilometre run) is obtained from equation:

$$
L=\frac{D}{D_{\text {lim }}} \cdot L_{m}
$$

Table 6 provides predicted fatigue life for the service stress spectra and the spectra measured on the test polygon.

The ratio of these predicted fatigue life is an estimation of the acceleration (shortening) of the driving fatigue test that could be achieved on the test polygon compared to normal vehicle operation.

Table 6. Shape parameter of all measured stress spectra.

\begin{tabular}{|c|c|c|c|}
\hline Empty vehicle & $\begin{array}{c}\text { Real } \\
\text { operation }\end{array}$ & $\begin{array}{c}\text { Testing } \\
\text { ground }\end{array}$ & Acceleration \\
\hline $\mathrm{L}(\mathrm{km})$ & $3,178,000$ & 203,000 & 15.7 \\
\hline
\end{tabular}

\begin{tabular}{|c|c|c|c|}
\hline Loaded vehicle & $\begin{array}{c}\text { Real } \\
\text { operation }\end{array}$ & $\begin{array}{c}\text { Testing } \\
\text { ground }\end{array}$ & Acceleration \\
\hline L $(\mathrm{km})$ & $4,309,000$ & 337,000 & 12.8 \\
\hline
\end{tabular}

\section{Conclusions}

Several interesting conclusions and remarks can be formulated on the basis of this case study.

It has been verified that the parameter $s=1$ can be selected to generate the design stresses spectra in the form (1). This conclusion applies only to the bodywork nodes! For vehicle components, such as axles, this value may be different, because the driving manoeuvres are more important for their stresses.

The estimation of the other parameters of the design stress spectra $\left(H_{t o t}, \sigma_{a \max }\right.$ and $\left.H_{\max }\right)$ is a little more problematic, but the procedure proposed in chapter 3.1 is acceptable. In our case, it proved to be sufficiently conservative, which is essential at the stage of the initial design of a vehicle. 
Based on the demonstrated methodology (using fatigue damage calculations), it is possible to determine the acceleration of the driving fatigue tests on the testing grounds. A changing the composition of the test route can result in a change in the acceleration of this test. However, the composition of the test track cannot be any. For example, an intensified driving on special roads could cause an undesirable overheating of shock absorbers.

In the presented case study, the accelerations of the driving fatigue test were calculated separately for a city operation of an empty and fully loaded vehicle. In another practical case it would be possible to determine a more detailed mix of operating conditions.

In the presented case study, the acceleration of the driving fatigue tests were exemplarily determined for the mean fatigue life (because the mean S-N curve with $50 \%$ survival probability was considered). For specific commercial solutions, the required operational reliability should be taken into account. The authors of this paper have published some examples of engineering applications of a probabilistic approach to the fatigue life calculations $[5,6]$.

The contribution has been prepared under project LO1502 'Development of the Regional Technological Institute' under the auspices of the National Sustainability Programme I of the Ministry of Education of the Czech Republic aimed to support research, experimental development and innovation.

\section{References}

1. V. Chmelko, M. Margetin: The methodology of transformation of the nominal loading process into a root of notch. Procedia Structural Integrity, Vol. 5, (2017), 825-831

2. M. Ruzicka, M. Hanke, M. Rost: Dynamic strength and fatigue life (University textbook, Czech Technical University, 1987, in Czech)

3. J. Neugebauer, V. Grubisic, G. Fischer: Procedure for Design Optimization and Durability Life Approval of Truck Axles and Axle Assemblies (SAE Technical Paper Series 892535, 1989)

4. M. Kepka: Durability and Fatigue Life Investigation of Bus and Trolleybus Structures: Review of SKODA VYZKUM Methodology. Proceedings of the 2nd International Conference on Material and Component Performance under Variable Amplitude Loading (VAL2009), DVM, Darmstadt, Germany, March 23 - 26, 2009, pp.1231-1240

5. M. Kepka, M. Kepka Jr.: Identification of Possible Causes of Fatigue Failure in Radius Rod Pins for Trolleybus Rear Axles using Parametric Calculations of Fatigue Life. Proceedings of 7th International Conference on Mechanics and Materials in Design (MCD2017), Albufeira/Portugal, June 11-15, 2017
6. M. Kepka, M. Kepka Jr.: Parametric calculations of fatigue life of critical part of trolleybus rear axle. Procedia Engineering, Vol. 213, (2018), 227-238 